\title{
Blind Spot Mitigation in Phased Array Antenna using Metamaterials
}

\author{
Thomas Crépin*, Cédric Martel, Benjamin Gabard, \\ Fabrice Boust \\ Onera - The French Aerospace Lab \\ F-31055, Toulouse, France \\ *corresponding author, E-mail: Thomas.Crepin@onera.fr \\ Jean-Paul Martinaud, Thierry Dousset \\ Thales Systèmes Aéroportés, F-78852, Elancourt, France \\ Pablo Rodriguez-Ulibarri, Miguel Beruete \\ Public University of Navarre, 31006, Pamplona, Spain
}

\author{
Claudius Loecker, Thomas Bertuch \\ Fraunhofer FHR, 53343, Wachtberg, Germany \\ José Antonio Marcotegui \\ Tafco Metawireless, 31110, Noáin, Spain \\ Stefano Maci \\ University of Siena, 53100, Siena, Italy
}

\begin{abstract}
In this work, a metaradome based on a fakir's bed of nails is designed and tested in order to suppress the blind directions of a 100-element antenna array. The antenna is a microstrip array designed to operate in X-band. The fakir's bed metamaterial-like was first approximated using analytical formulas before a full-wave numerical optimization. Experimental results are exposed and confronted to numerical results. They show a significant reduction of the blind spot subsequent to the metaradome addition.
\end{abstract}

Keywords—antenna array, metamaterial, scan blindness

\section{INTRODUCTION}

The blind directions are well known phenomena in antenna arrays. The physical effect is a mode interaction between the Bloch array eigenmodes and the propagating surface waves or leaky waves. The apparition of a dip (Fig. 1) is generally observed closed to the grating lobe excitation. The blind spot is associated with a specific scan angle according to the mode coupling and induce a strong mismatch at the input ports, which implies inability to send and receive signals in this space angle region. This array constraint has been discussed by many authors [1] [2] but a similar phenomenon has been early described in optical reflector arrays [3] and was identified as Wood's anomalies.

The present article presents some results from the METALESA project (EDA funded project on the application of metamaterials to active antenna arrays). We focused on the work package dedicated to study how metamaterials could bring solutions to cancel the blind directions and the corresponding mismatches. Two ways were considered:

- The design of metamaterials or EBG between the unit cells corresponding to the single radiating elements in order to make the surface waves (SW) propagation forbidden.

- The manufacturing of an anisotropic radome placed in the near field zone to achieve a wide angle impedance matching (WAIM) [4]
In this paper, only the first approach will be developed. The first part is dedicated to the problem description. Then, a first design able to make the SWs forbidden is proposed. Finally, the characterizations are shown and discussed.

\section{SCAN BLINDNESS}

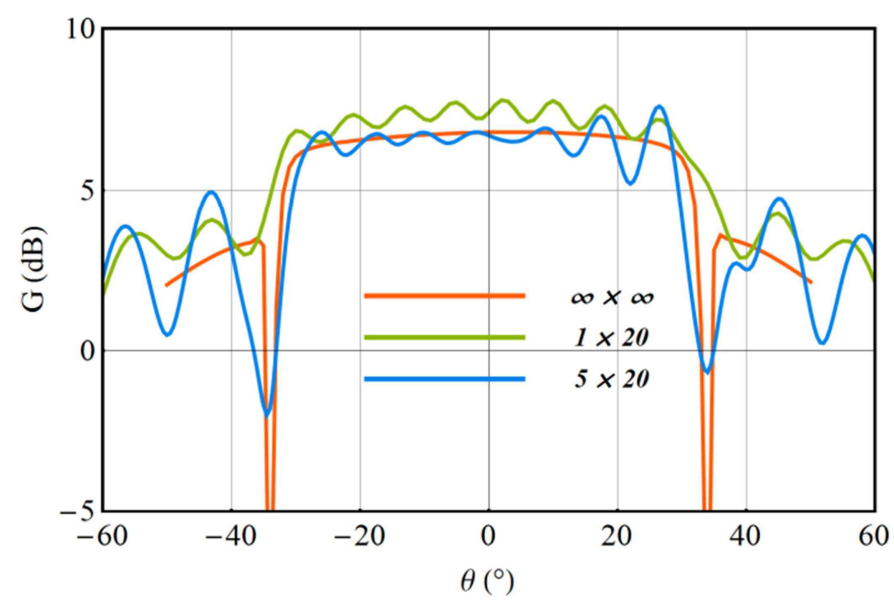

Fig. 1. Elementary radiation pattern, for an infinite array, a 20 elements single line (element \#10) and a 5x20 elements array (element \#50).

During the project, the array periodicity was set to $19 \mathrm{~mm} \mathrm{x}$ $19 \mathrm{~mm}$ (0.63 wavelengths at the center frequency). Each radiating element is composed of a cavity including two stacked patches. The top one is passive and the bottom one is connected to a coaxial connector probe.

According to this geometry, the blind direction can be identified through a full wave EM simulation of an infinite array.

The array simulated at $10 \mathrm{GHz}$ shows a scanning angle that corresponds to a blind spot closed to $34.5^{\circ}$ in the E-plane. We can also notice that the blind spot requires at least 5 rows to be 
clearly observed (Fig. 1). Around the blind spot angle, close to the antenna, the simulated Poynting vector is mainly parallel to the $x$-axis. The wave mode then presents all the characteristics of a TM surface wave. Moving away from the array, as this surface wave decreases, the propagation direction becomes more oriented in the expected direction.

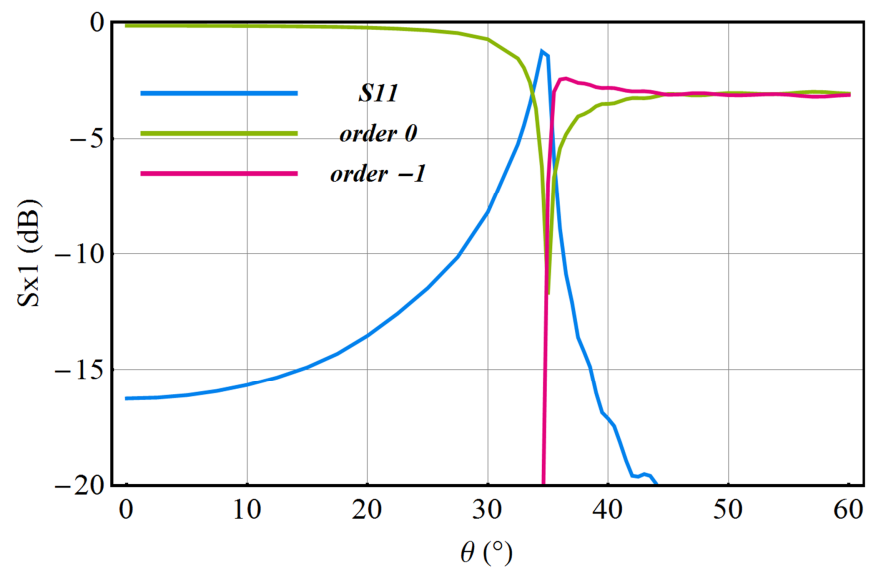

Fig. 2. S11 (return loss) for an infinite array, compared to the transmission level of the main lobe ( 0 order) and of the grating lobe ( -1 order).

Fig. 2 exhibits the reflection calculated at the wave port (S11) and the radiated energy into the grating orders 0 and -1 (simulation of an infinite array achieved with COMSOL Multiphysics () . S11 tends toward $0 \mathrm{~dB}$ close to the blind direction while a strong deep appears in the transmission of the 0 order array mode. Beyond the resonance, the -1 order transmission rises corresponding to the grating lobe.

During the study, we focused on the attenuation of the TM surface wave for the $9-11 \mathrm{GHz}$ bandwidth. Two additional goals were added to the study:

- Not to degrade the antenna efficiency for frequency and incidences far from the blind spot.

- Not to modify the design of the radiating element.

\section{METARADOME DESIGN}

\section{A. First approach: the fakir's bed metamaterial}

Many metamaterials and EBG are able to make SWs forbidden. Most examples are based on planar technologies like split ring resonators or high impedance surfaces (HIS) which are very useful to reduce the electromagnetic coupling between for example microstrip antennas or radiating apertures.

Despite the large number of technologies, the wide bandwidth constraint imposes to use of an electromagnetic band gap preferably a narrowband metamaterial due to a resonance phenomenon (e.g. HIS). Actually, to obtain the right metaradome properties we had had to combine both effects. Moreover a geometrical constraint had to be taken into account due to the small spacing between the radiating elements. Indeed, the $19 \mathrm{~mm}$ periodicity combined to the cavity width leaves only $7 \mathrm{~mm}$ free to introduce the artificial media. Accordingly to these dimensions, the fakir's bed of nails was chosen [5]. This structure is compatible with the layout and consists of metallic pins interconnected by a metal plane. A homogenization model has been proposed in the literature [6, 7] which gives the characteristics of the modes bounded by this metamaterial and as well as the $k_{x}$ wavenumber evolution in relation to the geometrical variables. The homogenization model gives two indications which were used to optimize the metaradome (cf. Fig. 3):

- The pin length $L$ determines the stop band frequency $\left(f \geq \frac{c}{4 L \sqrt{\varepsilon_{r}}}\right)$,

- To maximize the value of $\left|k_{x}\right|$ for frequencies close to the stop band limit, the effective plasma frequency has to be increased. This plasma frequency can be estimated using $f_{p}=\frac{c}{a \sqrt{2 \pi \varepsilon_{r}}}\left(\ln \left(\frac{a}{2 \pi r}\right)+0.525\right)^{-1 / 2}[8]$ with $r$ the radius and $a$ the spacing.

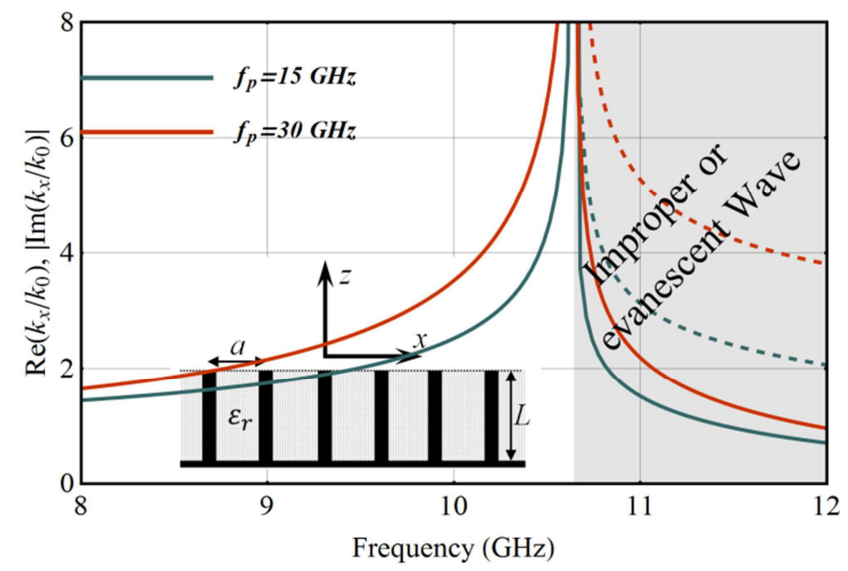

Fig. 3. Frequency evolution of the x-component of a TM SW which propagates along a fakir's bed of nails (pins length $\mathrm{L}=4.75 \mathrm{~mm}$ )

\section{B. Metamaterial optimization}

Fig. 4 exhibits the final metaradome unit cell optimized by ANSYS HFSS $®$. The metamaterial consists of a double pin rows parallel to the $y$-axis in order to cancel propagation along $x$-axis. In other words the layout was optimized to maximize the scanning angles while maintaining a good impedance matching in the whole frequency range.

To obtain significant results, different parametric computations were necessary. Geometric choices result from these studies in relation with fabrication constraints.

The length of the pins and their radius have been respectively reduced to $L=4.75 \mathrm{~mm}$ and $r=0.2 \mathrm{~mm}$. To increase the plasma frequency $f_{p}$ approximately up to $30 \mathrm{GHz}$ the distance between the pins has been fixed to $a=2.38 \mathrm{~mm}$. Consequently the stop band frequency is close to $10.6 \mathrm{GHz}$ which means that the band gap effect is combined with a resonance phenomenon. Accordingly to the wide band impedance matching constraint, we have chosen a low permittivity substrates $\left(\varepsilon_{\mathrm{r}}=2.2\right)$. Furthermore, to improve the matching, one extra-superstrate has been added on the multilayer (1.57 $\mathrm{mm}$ thick). 
a)
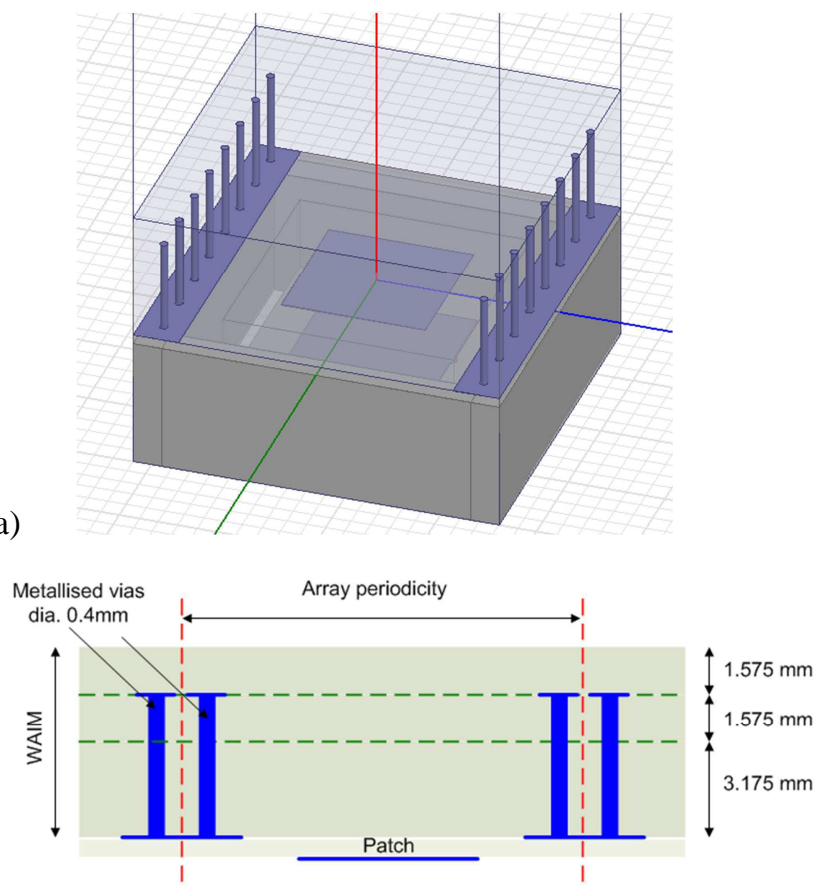

b)

Fig. 4. a) Unit cell with the metaradome. b) Details of the metaradome structure.

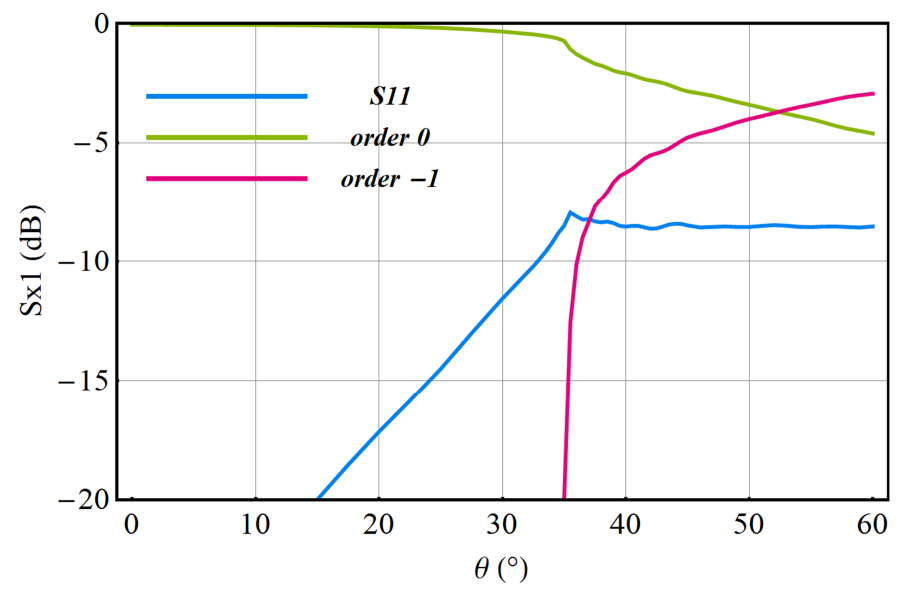

Fig. 5. S11, main lobe (order 0) and grating lobe (order -1) for an infinite array covered by the metaradome.

\section{MEASUREMENT RESULTS}
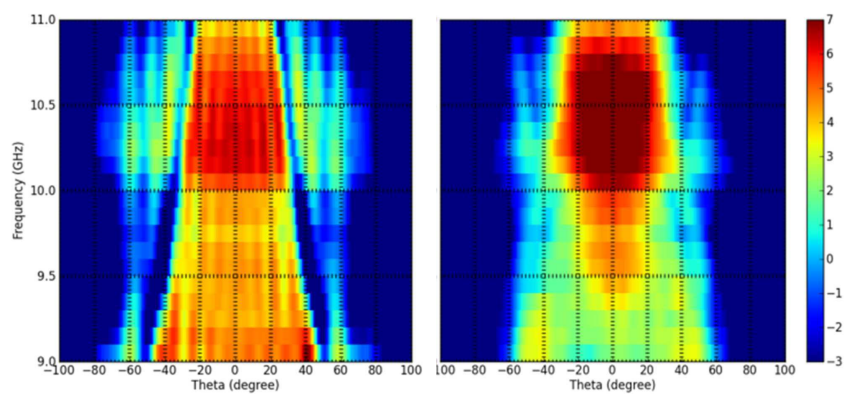

Fig. 6. E-plane embedded radiation patterns of the central element (without the metaradome and with the metaradome).
To measure radiation patterns and the array gains (with and without metaradome), the antenna was disposed in an anechoic chamber.

Each radiating element was measured between 9 and $11 \mathrm{GHz}$. After that, the total radiation pattern was obtained combining these results. Each measurement was done by connecting the element to a VNA while the others were terminated by $50 \Omega$ loads in order to include the real coupling effects.

Fig. 6 shows the radiation pattern of the central element. Without the metaradome, the blind direction can be clearly identified by the two low-level symmetrical lines across the angular-frequency space. Adding the metaradome, the blind directions are significantly mitigated. Furthermore the gain has slightly been increased at frequencies over $10 \mathrm{GHz}$.

Due to a measurement technique that includes the array environment of each radiating element, the complete antenna pattern can be calculated by summing the 100 radiation pattern. Figure 7 shows a comparison, at $10 \mathrm{GHz}$, between the array measured radiation pattern with and without metaradome, for different scan angles. The WAIM metaradome clearly removes the blind spot close to $35^{\circ}$ scan angle. The measured gain of the array with the metaradome is $23.8 \mathrm{dBi}(25.3 \mathrm{dBi}$ without metaradome), at $10 \mathrm{GHz}$ for the broadside configuration. It can be also noted that the grating lobe has decreased for scan angles between $35^{\circ}$ and $40^{\circ}$.

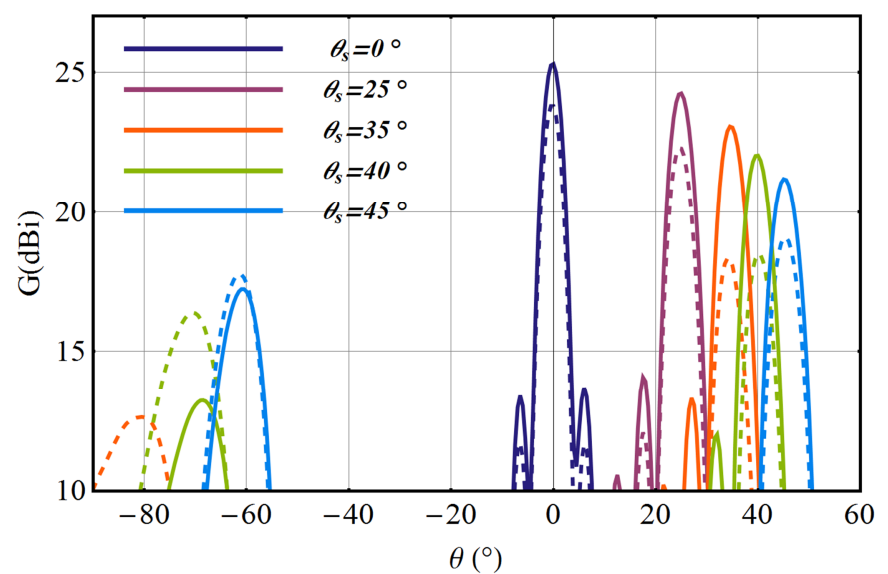

Fig. 7. Array radiation pattern at $10 \mathrm{GHz}$ with (solid lines) and without metaradome (dashed lines).

\section{CONCLUSION}

During this study a 5 x 20 antenna array in X-band $\left(0.63 \lambda_{0}\right.$ spacing) has been fabricated and characterized. To cancel the blind spot a WAIM metaradome was located on the structure.

We observed a significant reduction of the phenomenon. The grating lobes were mitigated $\left(10 \mathrm{~dB}\right.$ for a $135^{\circ}$ phase shift $)$ and the gain was increased at broadside (approximately $1.5 \mathrm{~dB}$ ). The antenna array was able to scan up to $55^{\circ}$ from broadside, with $1 \mathrm{GHz}$ bandwidth. 
However, negative points have to be pointed out. The gain decreased for frequency below $10 \mathrm{GHz}$. Furthermore, degradations of the radiation patterns have been observed for H-plan scanning (but this issue was not addressed in the present study).

Further improvements may be achieved by proceeding optimizations of the radiating element (patch size and cavity dimensions) combined with the metaradome design. The introduction of metamaterial structures in the H-plan could also extend the antenna capabilities.

These results could be particularly interesting for new generation radars and active antennas.

\section{ACKNOWLEDGEMENTS}

This work was supported by the METALESA project A1089-RT-GC that is coordinated by the European Defence Agency (EDA) and funded by 11 contributing Members (Cyprus, France, Germany, Greece, Hungary, Italy, Norway, Poland, Slovakia, Slovenia and Spain) in the framework of the Joint Investment Programme on Innovative Concepts and Emerging Technologies (JIP-ICET).

\section{REFERENCES}

[1] G.H. Knittel, A. Hessel and A. Oliner, Element pattern nulls in phased arrays and their relation to guided waves, Proceedings of the IEEE, vol. 56, nº111, pp. 1822-1836, 1968.

[2] D.M. Pozar and D. Schaubert, Scan blindness in infinite phased arrays of printed dipoles, Antennas and Propagation, IEEE Transactions on, vol. $32, n^{\circ} 16$, pp. 602-610, 1984.

[3] A. Hessel and A.A. Oliner, A new theory of Wood's anomalies on optical gratings, Appl. Opt. vol 4, no 10, 1975-1297, 1965.

[4] P. Rodriguez-Ulibarri, M. Beruete, F. Falcone, T. Crépin, C. Martel, F. Boust, C. Loecker, K. Herbertz, C. Salzburg, T. Bertuch, J.P. Martinaud, T. Dousset, J. A. Marcotegui, Metaradome for Blind Spot Mitigation in Phased-Array Antennas, submitted to EuCAP 2014 conference.

[5] R.J. King, D.V. Thiel and K.S. Park, The synthesis of surface reactance using an artificial dielectric, Antennas and Propagation, IEEE Transactions on, vol. 31, no3, pp 471-476, 1983

[6] M.G. Silveirinha, C.A. Fernandes and J.R. Costa, Electromagnetic Characterization of Textured Surfaces Formed by Metallic Pins, Antennas and Propagation, IEEE Transactions on, vol. 56, no2, pp 405415, 2008.

[7] A.B. Yakovlev, M.G. Silveirinha, O. Luukkonen, C.R. Simovski, I.S. Nefedov and S.A. Tretyakov, Characterization of Surface-Wave and Leaky-Wave Propagation on Wire-Medium Slabs and Mushroom Structures Based on Local and Nonlocal Homogenization Models, Microwave Theory and Techniques, IEEE Transactions on, vol. 57, no11, pp 2700-2714, 2009.

[8] S. A. Tretyakov, Analytical Modeling in Applied Electromagnetics, Boston, MA: Artech House. 